\title{
A Simple PCR Cloning Step to Utilize a Binary Vector for Identification and Characterization of Enhancer Element in Plant Promoters
}

\author{
Dipnarayan Saha • Vajinder Kumar • \\ Sripad Ramachandran Bhat $\cdot$ Ramamurthy Srinivasan
}

Received: 16 December 2011/ Accepted: 14 April 2012/Published online: 31 May 2012

(C) NAAS (National Academy of Agricultural Sciences) 2012

\begin{abstract}
Identification and characterization of regulatory elements, such as enhancers, require designing of specialized plant transformation vector to testify its unique features of position and orientation-independent core-promoter activation. Very few plant transformation vectors are available till date for identification of enhancer elements in plant promoters. Hence, we need to utilize the existing binary plant transformation vectors, such as pBI101, through tedious and timeconsuming restriction enzyme-based cloning steps. We describe here a simple one-step PCR-based cloning strategy to introduce a 35S CaMV minimal promoter in a binary plant transformation vector pBI101, and utilize the unique features to identify a lateral organ junction tissue-specific enhancer element in a plant promoter from Arabidopsis thaliana.
\end{abstract}

Keywords Arabidopsis $\cdot$ Enhancer detection vector $\cdot$ Enhancer-like activity $\cdot$ Minimal promoter $\cdot$ Plant promoter

\section{Introduction}

Conventional and functional genomics-based promoter identification strategies, especially the T-DNA-based promoter tagging, has made immense contribution toward isolation and characterization of number of promoter elements in plants [9, 23, 27]. Characterization of plant promoters is crucial and prerequisite toward understanding the regulation specificity of promoters and their appropriated utilizations in crop improvement through genetic engineering $[19,20]$. Characterization of promoter essentially involves functional analysis of the inherent regulatory elements responsible for imparting regulation specificity. Promoter deletion, linker scanning, and base substitution

D. Saha $\cdot$ V. Kumar $\cdot$ S. R. Bhat $\cdot$ R. Srinivasan $(\bowtie)$ National Research Centre on Plant Biotechnology, Indian Agricultural Research Institute, New Delhi 110 012, India e-mail: srinivasan53@gmail.com; sri@iari.res.in

D. Saha

National Research Centre on DNA Finger Printing, National Bureau of Plant Genetic Resources, New Delhi 110 012, India approaches are few widely adopted strategies for identifying regulatory sequences in promoters [27]. The ability and specificity of the promoter element in driving reporter gene expression pattern provides clues about the possible regulatory role played by the promoter [27]. Thus, the choice of plant transformation vector is very crucial in designing appropriate vector construct for functional analysis of a promoter.

The transcriptional enhancers are important regulatory elements involved in regulation of several developmentally regulated genes from position and orientation independent locations in a genome [3, 4, 13, 18, 25, 29]. Enhancer elements are responsible for imparting spatial, temporal, and environmentally regulated gene expression in plants. However, majority of the enhancer elements require support from cognate core-promoter sequence for its regulatory function $[5,25]$. Thus, harnessing the above typical features, the enhancer-like activity of a promoter sequence can be detected only through a vector having a reporter gene driven by a minimal promoter. In eukaryotes, such as mammalian system and Drosophila, enhancer testing vectors have been developed $[2,22,26]$. Although several binary plant transformation vectors are available and utilized for functional characterization of cis-regulatory 
elements in plant promoters, the in vivo detection and validation of enhancer element remains a significant challenge. Here, unlike the tedious restriction enzyme-based modification, we report a simple PCR cloning step in a popular plant transformation vector $\mathrm{pBI}-101$ to use it as a tool for identification and characterization of enhancer element in plant promoters. The utility of the strategy to clone vector construct was demonstrated in identification of a tissue-specific enhancer element in the LOJ gene promoter, specific for regulating expression of GUS gene in the lateral organ junction tissues of Arabidopsis thaliana.

\section{Materials and Methods}

Plant Material and Growth Conditions

Wild type Arabidopsis ecotype Columbia (Col-0) was used in the present study. The seeds of Arabidopsis were germinated on $0.8 \%$ agar-gelled Murashige and Skoog's (MS) medium as per Feldmann and Marks [11]. The seedlings were maintained in culture room at $22 \pm 1{ }^{\circ} \mathrm{C}$ and $16 / 8 \mathrm{~h}$ photoperiod $\left(150 \mu \mathrm{mol} \mathrm{m} \mathrm{m}^{-2} \mathrm{~s}^{-1}\right)$. Germinated seedlings of 12-14 days old were transferred to plastic pots filled with soilrite and grown at $23 \pm 1{ }^{\circ} \mathrm{C}$ under long day conditions in a glasshouse [8]. The plants were irrigated with Hoagland's solution and water on alternate days, respectively.
Cloning of Minimal Promoter in pBI101

A binary plant transformation vector pBI-101 $(\sim 12.2 \mathrm{~kb})$ [14] consisting of a multiple cloning site (MCS), a promoter-less reporter gene uidA within the T-DNA sequence and a plant and bacterial selectable marker gene nptII was used to clone a minimal promoter sequence and utilize as an enhancer testing vector pBI-MP1. An 83-bp minimal promoter sequence of the $35 \mathrm{~S} \mathrm{CaMV}$ promoter $(-88$ to -6) was PCR amplified from plasmid pBinAR [12] using sequence-specific primers (Table 1) and was ligated into the SalI-BamHI site of the MCS region upstream of uidA gene of pBI101 vector to generate pBI-MP1.

To test enhancer-like activity of a promoter fragment through re-designed pBI-MP1 vector construct, a 460-bp (-673 to -214) 5'-upstream DNA sequence of the At2g39230.1 gene of Arabidopsis, known to confer lateral organ junction tissue-specific gene expression [21] was amplified by PCR using sequence-specific primers (Table 1) and cloned into the EcoRI site of the pBI-MP1 to designate pBI-LPEn4. The EcoRI site is situated $\sim 2 \mathrm{~kb}$ downstream of the ATG start codon of uidA reporter gene and $35 \mathrm{~S}$ CaMV minimal promoter. Both the vector constructs (pBI-MP1 and pBI-LPEn4) generated above were sequence verified. The binary vector $\mathrm{pBI}-121$ containing the uidA reporter gene driven by full length $35 \mathrm{~S}$ CaMV promoter [7] was used in the study for comparison of reporter gene expression patterns. All the above vectors were transformed into Agrobacterium strain GV3101 [15]

Table 1 Nucleotide sequences of the primers used in the present study (underlined sequences are restriction enzyme recognition sequences added to the primers for facilitating directional cloning vectors)

\begin{tabular}{|c|c|c|}
\hline Sl. No. & Primers & Sequences $\left(5^{\prime}-3^{\prime}\right)$ \\
\hline \multicolumn{3}{|c|}{ Primers used for cloning of $35 \mathrm{~S}$ CaMV minimal promoter } \\
\hline 1. & 35SCaMVmp F: & 5'-GCGTCGACCACTGACGTAAGGGATGAC-3' \\
\hline 2. & 35SCaMVmp R: & 5'-CGGGATCCTCTCCAAATGAAATGAACTTC-3' \\
\hline \multicolumn{3}{|c|}{ Primers used for cloning of LOJ promoter } \\
\hline 3. & -673 lojRO For: & 5'-AGTGTCTACTATTCTAATAAGCCCA-3' \\
\hline 4. & -214 lojRO Rev: & 5'-CAACAGTATAAATTCAACTCCCAC- $3^{\prime}$ \\
\hline \multicolumn{3}{|c|}{ Primers for confirmation of presence of T-DNA in transgenic Arabidopsis } \\
\hline 5. & Gus $1022 \mathrm{~F}$ : & 5'-CAGATGAACATGGCATCGTG-3' \\
\hline 6. & GUS $75 \mathrm{~F}$ : & 5'-CGAAAACTGTGGAATTGATC-3' \\
\hline 7. & GUS 506 R: & 5'-CCGGCATAGTTAAAGAAATC-3' \\
\hline \multicolumn{3}{|c|}{ Primers for detection of uidA gene transcript in $R T-P C R$ analysis } \\
\hline 8. & GUS For: & 5'-CCATTTGAAGCCGATGTCACGCC-3' \\
\hline 9. & GUS Rev: & 5'-TATCGGTGTGAGCGTCGCAGAAC-3' \\
\hline \multicolumn{3}{|c|}{ Primers for relative quantification in $q R T-P C R$ analysis } \\
\hline 10. & GUS F 101 & 5'-GGTGGGAAAGCGCGTTACAAG \\
\hline 11. & GUS R 265 & 5'-TTGCCGTAATGAGTGACCGCA \\
\hline 12. & UBQ 10 Fwd & 5'-GGCCTTGTATAATCCCTGATGAATAAG \\
\hline 13. & UBQ $10 \mathrm{Rev}$ & 5'-AAAGAGATAACAGGAACGGAAACATAGT \\
\hline
\end{tabular}


by freeze and thaw method before floral dip transformation in Arabidopsis.

Plant Transformation and PCR Confirmation of Transgenic Plants

About 40-45-days-old wild type Arabidopsis plants bearing maximum unopened flower buds were used for in planta plant transformation by floral dip protocol as per the Clough and Bent [8]. Mature seeds from the transformed $\left(\mathrm{T}_{0}\right)$ plants were harvested and selected on MS medium consisting of kanamycin $\left(50 \mathrm{mg}^{-1}\right)$ during germination process as described above. The transgenic plants $\left(\mathrm{T}_{1}\right)$ were allowed for self pollination and $\mathrm{T}_{2}$ seeds were collected separately from individual $\mathrm{T}_{1}$ plants.

Total genomic DNA was extracted from $\mathrm{T}_{1}$ and $\mathrm{T}_{2}$ plants by cetyltrimethylammonium bromide (CTAB) method [10]. Approximately $50 \mathrm{ng}$ of genomic DNA from each plant was used as a template for PCR analysis. The uidA gene-specific primers and primers spanning uidA gene and cloned DNA were used to detect the presence of T-DNA in $\mathrm{T}_{2}$ plants (Table 1).

\section{Histochemical GUS Assay and RT-PCR Analysis}

The histochemical GUS analysis of the transgenic lines were performed using 5-bromo-4-chloro-3-indolyl- $\beta$-glucuronic acid (X-gluc) as a substrate as described by Jefferson et al. [14]. The plant tissues were soaked in GUS assay buffer, vacuum infiltrated for $5 \mathrm{~min}$ and incubated at $37^{\circ} \mathrm{C}$ for overnight under dark conditions. The chlorophyll from the tissues was cleared by twice changing of $70 \%$ ethanol and incubating at $37{ }^{\circ} \mathrm{C}$. The GUS expression was observed under the Nikon HFX II light stereomicroscope and photographed using digital camera.

Total RNA was extracted from lateral organ junction enriched tissues of all the $T_{2}$ plants using TRI REAGENT ${ }^{\circledR}$ (Molecular Research Center (MRC) Inc. as per the manufacturer's protocol and quantified using NanoDrop (ND1000) spectrophotometer. The purity of RNA was estimated by spectrophotometric measurement of absorbance at $\mathrm{A}_{260} / \mathrm{A}_{280}$ ratio and visually by analyzing the integrity of $18 \mathrm{~S}$ and $28 \mathrm{~S}$ rRNA bands on $1.2 \%$ denaturing grade formaldehyde agarose gel. Equalized amount of RNA samples were treated with RNase free-DNaseI and used as template for RT-PCR analysis using One Step RT-PCR kit (QIAGEN) as per the manufacturer's protocol. The uidA gene-specific primers GUS $75 \mathrm{~F}$ and GUS $506 \mathrm{R}$ were used to detect the presence of GUS transcript. The presence of Actin8 gene transcript as control in all the RNA samples were analyzed using primers ACT8 $\mathrm{F}$ and ACT8 R (Table 1). Quantitative real-time reverse transcription PCR (qRT-PCR) was used for relative quantification of GUS transcript in the lateral organ junction tissues of the transgenic plants. A total of $1.0 \mu \mathrm{g}$ of total RNA (DNAfree) extracted from the lateral organ junction tissue enriched samples was converted to single standard cDNA using Superscript III reverse transcriptase (Invitrogen Inc.) followed by dilution to $20 \mathrm{ng} / \mu \mathrm{l}$. A total $10-\mu \mathrm{l}$ reaction volume consisting of SYBR FAST qRT-PCR Master Mix $(2 \times)$ Universal (KAPA Biosystems) and 10 pmol of each primers for $G U S$ and $U B Q 10$ (Ubiquitin) specific transcripts (Table 1) were used in all the qRT-PCR reactions. The primers were designed using conditions set for $50 \%$ GC content, 59-61 ${ }^{\circ} \mathrm{C}$ Tm, 20-22 nt, and 100-200-bp product size using the primer designing option of IDT (http://eu.idtdna.com/scitools/Applications/RealTimePCR). Normalization between samples was carried out with $U B Q$ $(U B Q 10)$ as reference gene. Each sample was replicated thrice in the qRT-PCR reaction and control reactions without template were used to minimize contaminations. The qRT-PCR reactions were performed on a ROCHE LightCycler 480 system using the following thermal cycling programme (40 cycles): $3 \mathrm{~min}$ at $95{ }^{\circ} \mathrm{C}$ (enzyme activation), $3 \mathrm{~s}$ at $95{ }^{\circ} \mathrm{C}$ (denaturation), and $30 \mathrm{~s}$ at $60^{\circ} \mathrm{C}$ (annealing/extension) which includes data acquisition period. Finally, a dissociation curve analysis was performed from 65 to $95{ }^{\circ} \mathrm{C}$ in increments of $0.5{ }^{\circ} \mathrm{C}$, each lasting for $5 \mathrm{~s}$, to confirm the presence of a specific product. Fold change in expression values were calculated by the $2^{-\Delta \Delta \mathrm{C}_{\mathrm{T}}}$ method [16].

\section{Results}

Features of pBI-MP1 Vector for Enhancer Characterization

The vector pBI-MP1 generated by us in the present study was designed for detection and analysis of enhancer elements and suppressors in the isolated plant promoters (Fig. 1a). The vector was generated utilizing the pBI-101 backbone and taking the advantage of its basic components, such as promoterless reporter gene uidA, which is unable to express unless linked with a promoter element, and the plant selectable marker gene $n p t I I$ for transformant selection. Since an enhancer element requires a support from core or minimal promoter sequence for its activity, we have introduced 83-bp minimal promoter sequence ( -88 to -6 ), consisting only TATA box sequence, from the 35S CaMV promoter by simple PCR cloning step, instead of the laborious restriction enzyme digestion approach. The minimal promoter sequence was PCR amplified from pBinAR plasmid and cloned into the SalI/BamHI site of MCS region of pBI-101. The vector construct was confirmed through digestion with SalI and BamHI restriction 
a

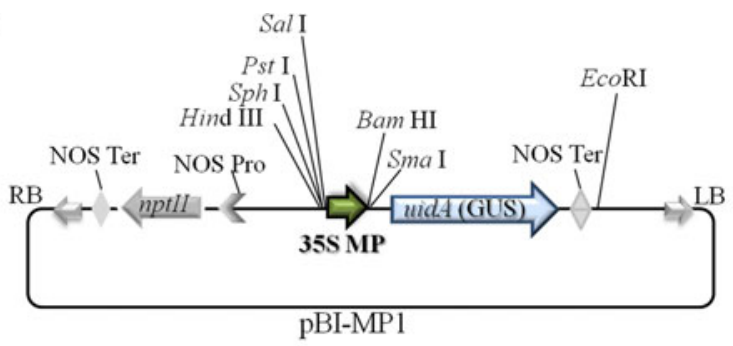

b

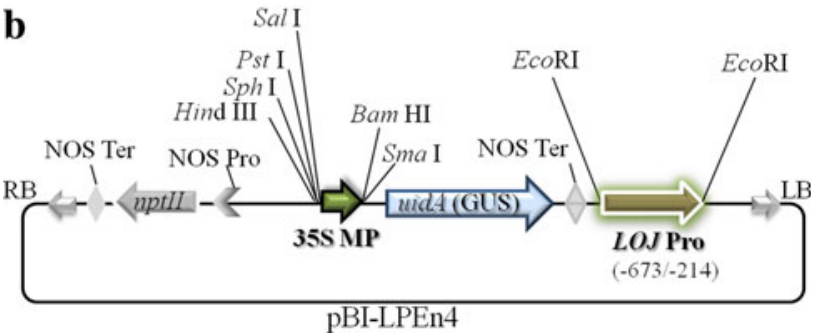

Fig. 1 Schematic diagram of the vector constructs. a The features in the T-DNA region of the pBI-MP1 vector are depicted in the map. An 83-bp minimal promoter (designated as $35 \mathrm{~S} \mathrm{MP)} \mathrm{from} 35 \mathrm{~S} \mathrm{CaMV}$ promoter containing only TATA sequence is cloned in the Sal1BamHI site of the promoterless pBI-101 vector backbone. b T-DNA features of the pBI-LPEn4 construct showing the $35 \mathrm{~S}$ minimal promoter (35S MP) upstream to uidA gene and the $5^{\prime}$ upstream DNA sequence of the LOJ gene (designated as LOJ Pro) cloned in the EcoRI site downstream to uidA gene

enzymes (RE), which released approximately 83-bp fragment followed by sequencing of the vector to confirm the cloned minimal promoter sequence.

To demonstrate the efficacy of the vector, a $5^{\prime}$ upstream sequence of the At2g39230.1 (LOJ) gene ( -673 to -214 ), previously identified by us to confer lateral organ junction tissue-specific expression [21], was cloned in the EcoRI site of the pBI-MP1 vector and verified through RE digestion and sequencing (Fig. 1b). Cloning of the LOJ promoter in the EcoRI site of pBI-MP1 resulted into positioning of the promoter to be tested in a distance more than $2 \mathrm{~kb}$ downstream from the ATG start codon and 35S minimal promoter. The cloning in EcoRI site also resulted into clones with $L O J$ promoter in both sense and reverse orientation. Since the ability of the LOJ promoter fragment to drive reporter gene expression pattern in either orientation was observed, possibly due to the presence of the enhancer element [24], the clones with LOJ promoter fragment in sense orientation, with respect to its orientation toward +1 start site (TSS) was chosen for further transgenic studies. The resultant construct was designated as pBI-LPEn4 and was confirmed by RE digestions with EcoRI and sequencing. Thus, the features utilized in the above construct provided an optimal condition to detect the enhancer-like activity of a promoter element, which can function by activating a minimal promoter from a distantly and orientation independently in a construct.
Confirmation of Transgenic Plants

The transgenic Arabidopsis plants with the constructs pBIMP1, pBI-LPEn4, and native pBI-121 (containing full length 35S CaMV promoter) were generated through floral dip transformation. Seven transgenic plants each for both pBI-MP1 and pBI-LPEn4 and 19 transgenic plants of pBI121 were obtained from the above transformation. The $\mathrm{T}_{1}$ and $\mathrm{T}_{2}$ plants obtained by selecting on kanamycin plates were further analyzed for the presence of T-DNA through PCR. All the transgenic plants of each construct produced desirable amplicons for the presence of T-DNA using the primer sets to amplify GUS gene alone and DNA region spanning cloned fragment and GUS gene. The PCR confirmed plant lines were further selected for histochemical GUS analysis and expression analysis through RT-PCR.

\section{Histochemical GUS Analysis of Transgenic Plants}

The histochemical GUS analysis carried out in transgenic Arabidopsis plants with pBI-MP1 construct consisting of $35 \mathrm{~S}$ minimal promoter produced no visible GUS expression in any plant parts or tissues (Fig. 2a). However, the transgenic Arabidopsis plants consisting of pBI-LPEn4 construct, i.e., containing minimal promoter for driving uidA gene and the $5^{\prime}$ upstream sequence of LOJ gene cloned in the downstream of uidA gene, typically produced GUS expression in all the lateral organ junction tissues. As expected, the transgenic Arabidopsis plants harboring pBI121 construct produced constitutive GUS expression in all the plant organs due to the full length $35 \mathrm{~S} \mathrm{CaMV}$ promoter-driven GUS gene.

\section{GUS Expression Analysis by RT-PCR and qRT-PCR}

The transgenic Arabidopsis plants one each consisting of pBI-MP1, pBI-LPEn4, and pBI-121 constructs were further analyzed for the presence of GUS transcript through RTPCR analysis. The RNA samples from transgenic plants with pBI-LPEn4 and pBI-121 construct showed the presence of GUS transcript in abundance. Whereas, a very faint presence of GUS transcript was detected in the RNA samples from transgenic plant containing pBI-MP1 construct. In contrast, the GUS transcript was found totally absent in the RNA sample from the wild type Arabidopsis. Comparatively, the Act8 gene transcript was found consistent in RNA samples of all three transgenic and wild type Arabidopsis plants (Fig. 2b).

The relative quantification of GUS transcript in the lateral organ junction tissues by qRT-PCR with the ubiquitin mRNA as reference demonstrated that the level of GUS transcript in the lateral organ junction tissues increased by approximately 2.5 -fold (estimated through 

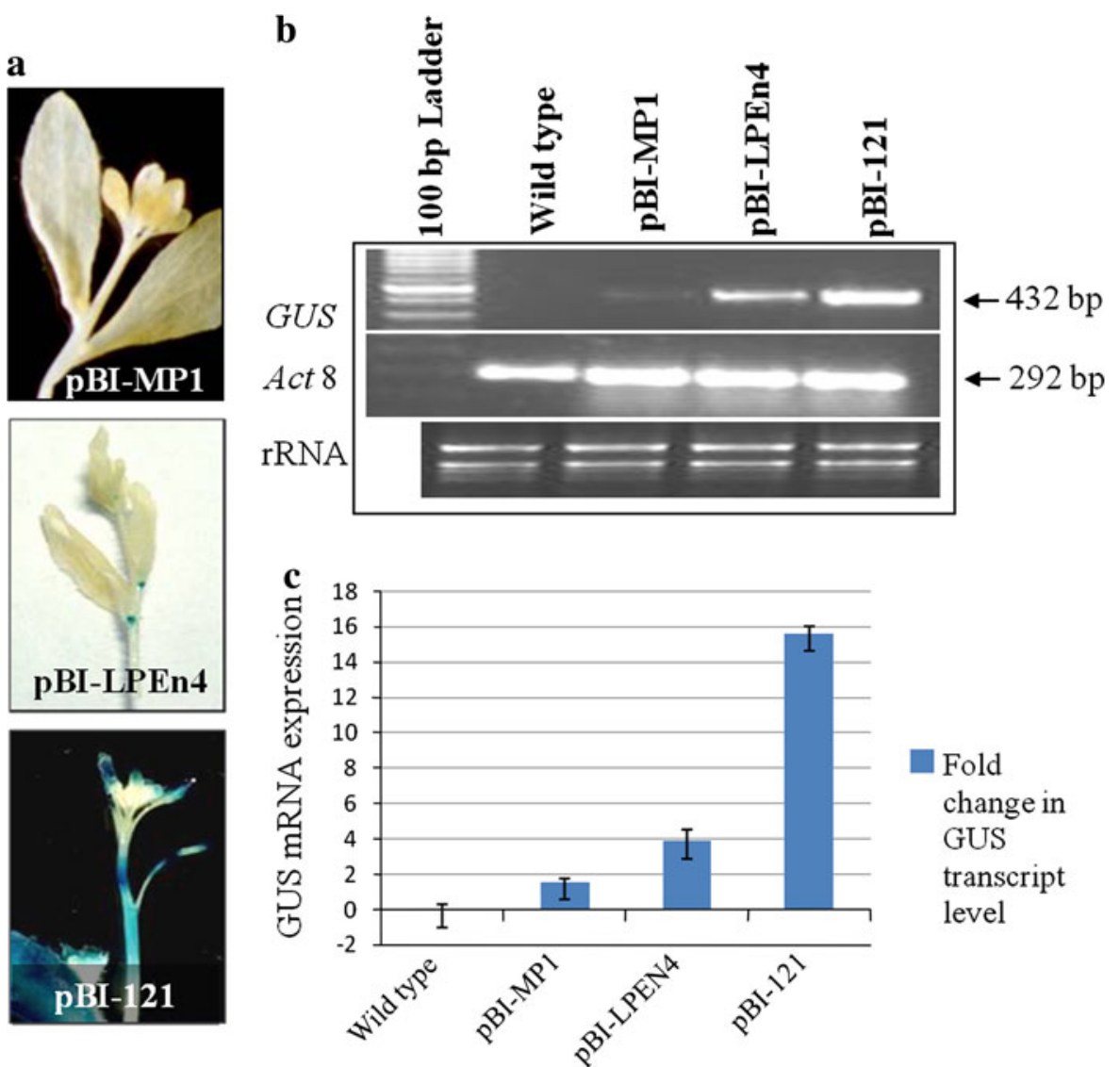

Fold

change in

GUS

transcript

level

Fig. 2 Histochemical GUS expression and analysis of GUS transcript in transgenic Arabidopsis plants. a Histochemical GUS expression pattern observed in transgenic Arabidopsis plants containing pBI-MP1, pBI-LPEn4, and pBI-121 constructs. No visible GUS expression observed in the junction tissues of plant containing pBIMP1. GUS expression was observed typically in all the lateral organ junction tissues of plant containing pBI-LPEn 4 construct. Constitutive GUS expression was observed in plants containing pBI-121 plasmid with full length $35 \mathrm{~S}$ CaMV promoter. b RT-PCR of RNA samples from $\mathrm{T}_{2}$ plants containing pBI-MP1, pBI-LPEn4, and pBI121 constructs. RNA from wild type was used as control. The upper

$2^{-\Delta \Delta \mathrm{C}_{\mathrm{T}}}$ value) in pBI-LPEn4 plant compared to the pBIMP1 plant (Fig. 2c). However, the GUS mRNA level in pBI-LPEn4 was found approximately fourfold less than the GUS mRNA level in pBI-121 plants with full length $35 \mathrm{~S}$ CaMV promoter (Fig. 2c). The GUS expression in wild type plant was used as non-transgenic control. The above RT-PCR and qRT-PCR studies corroborated with the histochemical GUS expression observed with the plants carrying respective vector constructs.

\section{Discussions}

Enhancer elements are among the regulatory elements of plant promoters which are particularly difficult to identify panel shows the presence of a prominent band for GUS transcripts (432 bp) in RNA from plants with pBI-LPEn4 and pBI-121 vector and a very faint band for GUS transcript in RNA from plant with pBIMP1. The middle panel shows the presence of Act 8 transcript in all the RNA samples including the wild type plant. The lower panel shows the integrity of the 28S and 18S rRNA bands in all the samples. c Relative quantification of GUS transcript in lateral organ junction tissues using real-time RT-PCR. Expression of GUS transcript is shown in relative (fold) to UBQ10 mRNA as reference and as a mean of three replications (noted by bars) \pm standard errors of the means

and annotate because of their ability to function even from a far off location and in either orientation [3, 13]. Both the above typical features are thus exploited as criteria for enhancer element identification in plant promoters. In addition, the ability to activate a minimal promoter for reporter gene expression by an enhancer element is also an important feature utilized to confirm enhancer-like activity of a plant promoter [5, 18, 25]. Therefore, an enhancer identification binary vector should essentially contain features like minimal promoter and cloning sites to position subject promoter fragment in suitable locations and orientations. Very few vectors are reported for plant promoter and enhancer testing in plants by utilizing truncated promoter sequence as minimal promoter from various sources like nos, $35 \mathrm{~S} \mathrm{CaMV}$, and Sesbania rostrata glb3 [28]. The 
minimal promoter sequence from $35 \mathrm{~S}$ CaMV promoter was also used in enhancer trap vectors or activation tag vectors for generation of mutants [6, 17].

For identification and characterization of enhancer element(s) in isolated novel plant promoters, we have made utilization of features of a binary plant transformation vector $\mathrm{pBI}-101$ to generate a vector $\mathrm{pBI}-\mathrm{MP} 1$ through a simple and rapid PCR cloning approach, which consists of a minimal promoter sequence from the $35 \mathrm{~S}$ CaMV promoter. The $83-b p$ ( -88 to -6$)$ minimal promoter sequence from 35S CaMV promoter consists of only TATA box and lacks the activator sequence as 1 [1, 6, 28] thus limiting its ability to confer visible reporter gene expression alone. The 35S CaMV minimal promoter used by Szabados et al. [28] was $-90 /+9$ to generate enhancer testing vector and by Campisi et al. [6] to generate enhancer trap vector was -60 CaMV 35S; both of these groups utilized cumbersome restriction enzyme digestion and cloning strategies. Whereas we carried out a very simple and rapid process of PCR amplification of the $-88 /-6$ region of the $35 \mathrm{~S} \mathrm{CaMV}$ promoter as the minimal promoter and ligated in the MCS region of the $\mathrm{pBI}-101$ to generate $\mathrm{pBI}-\mathrm{MP} 1$ vector. The $\mathrm{pBI}-\mathrm{MP} 1$ vector retains the promoter cloning advantages of pBI-101 in generating the promoter-reporter gene fusions. The unique restriction enzyme sites present in the MCS region allows cloning of single or concatenated promoter fragment upstream of the $35 \mathrm{~S}$ minimal promoter to identify enhancer-like activity through activation of the minimal promoter. While the EcoRI site downstream to uidA reporter gene and NOS terminator provides with an opportunity to clone a promoter fragment in distance and position independent manner.

The wild type Arabidopsis plants transformed with pBIMP1 could not produce any visible GUS expression in any tissues; although in RT-PCR and qRT-PCR studies negligible amount of GUS transcript was found in the RNA samples from transgenic plant for pBI-MP1 construct. This might be due to the minimal expression level directed by the TATA box, a characteristic feature of the minimal promoter, which otherwise was not sufficient enough to produce any visible GUS expression in tissues. In a proof of concept, the efficacy of the pBI-MP1 vector in detecting enhancer-like activity was demonstrated. The 460-bp LOJ promoter fragment, which was found to drive reporter gene expression in either orientation [24], when cloned in downstream of the uidA gene (pBI-LPEn4), it was able to activate the minimal promoter to confer visual GUS expression specifically in all the lateral organ junction tissues. Because positioning of the $L O J$ promoter DNA downstream to uidA gene at a distance of $\sim 2.0 \mathrm{~kb}$ eliminates the rarest possibility of transcriptional control by a regular promoter, the transcriptional activity of uidA gene owes to a regulatory element matching with the formal definition of an enhancer.
The pBI-MP1 vector generated here has been effectively used for detection of potential enhancer-like activity in a plant promoter. The plant promoters to be tested can be cloned in MCS region in either orientation in the $5^{\prime}$ upstream sequence of the uidA gene. In the present study, the cloning of plant promoter in the downstream of the uidA gene was facilitated by the unique EcoRI site, which may be replaced with another MCS region to provide users with more options. In our experiment, we have carried out plant transformation through floral dip in Arabidopsis, which rules out the misleading judgment of GUS expression by Agrobacterium. However, the uidA gene may be improved with intron containing-uidA gene to rule out the above limitation associated with Agrobacterium co-cultivation transformation. The uidA reporter gene can be replaced with $g f p$ to facilitate non-destructive reporter expression screening in transgenic plants. The vector modified to test the enhancer element in plant promoter thus would serve as a simple enhancer detection vector for functional analysis and characterization of enhancer or activator element(s) in Arabidopsis and other plant promoters.

Acknowledgments Authors acknowledge the help rendered by Dr K. V. Prabhu for growing Arabidopsis in National Phytotron Facility, IARI.

\section{References}

1. Benfey PN, Ren L, Chua NH (1990) Combinatorial and synergistic properties of CaMV 35S enhancer subdomains. EMBO J 9:1685-1696

2. Bessa J, Tena JJ, de la Calle-Mustienes E, Fernandez-Minan A, Naranjo S, Fernandez A, Montoliu L, Akalin A, Lenhard B, Casares F, Gomez-Skarmeta JL (2009) Zebrafish enhancer detection (ZED) vector: a new tool to facilitate transgenesis and the functional analysis of cis-regulatory regions in zebrafish. Dev Dyn 238:2409-2417

3. Blackwood EM, Kadonaga JT (1998) Going the distance: a current view of enhancer action. Science 281:60-63

4. Bulger M, Groudine M (2010) Enhancers: the abundance and function of regulatory sequences beyond promoters. Dev Biol 339:250-257

5. Butler JEF, Kadonaga JT (2001) Enhancer-promoter specificity mediated by DPE or TATA core promoter motifs. Genes Dev 15:2515-2519

6. Campisi L, Yang Y, Yi Y, Heilig E, Herman B, Cassista AJ, Allen DW, Xiang H, Jack T (1999) Generation of enhancer trap lines in Arabidopsis and characterization of expression patterns in the inflorescence. Plant J 17:699-707

7. Chen PY, Wang CK, Soong SC, To KY (2003) Complete sequence of the binary vector pBI121 and its application in cloning T-DNA insertion from transgenic plants. Mol Breed 11:287-293

8. Clough SJ, Bent AF (1998) Floral dip: a simplified method for Agrobacterium-mediated transformation of Arabidopsis thaliana. Plant J 16:735-743

9. Datla R, Anderson JW, Selvaraj G (1997) Plant promoters for transgene expression. Biotechnol Annu Rev 3:269-296 
10. Dellaporta SL, Wood J, Hicks JB (1983) A plant DNA minipreparation: version II. Plant Mol Biol Rep 1:19-21

11. Feldmann KA, Marks MD (1987) Agrobacterium-mediated transformation of germinating seeds of Arabidopsis thaliana: a non tissue culture approach. Mol Gen Genet 208:1-9

12. Hofgen R, Willmitzer L (1990) Biochemical and genetic analysis of different patatin isoforms expressed in various organs of potato (Solanum tuberosum). Plant Sci 66:221-230

13. Jin F, Li Y, Reb B, Natarajan R (2011) Enhancers-multidimensional signal integrators. Transcription 2:232-236

14. Jefferson RA, Kavanagh TA, Bevan MW (1987) GUS fusions: $\beta$-glucuronidase as a sensitive and versatile gene fusion marker in higher plants. EMBO J 6:3901-3907

15. Koncz C, Schell J (1986) The promoter of TL-DNA gene 5 controls the tissue-specific expression of chimeric genes carried by a novel type of Agrobacterium binary vector. Mol Gen Genet 204:383-396

16. Livak KJ, Schmittgen TD (2001) Analysis of relative gene expression data using real-time quantitative PCR and the $2^{-\Delta \Delta \mathrm{C}_{\mathrm{T}}}$ method. Methods 25:402-408

17. McBride KE, Summerfelt KR (1990) Improved binary vectors for Agrobacterium mediated plant transformation. Plant Mol Biol 14:269-276

18. Ong C-T, Corces VG (2011) Enhancer function: new insights into the regulation of tissue-specific gene expression. Nat Rev Genet 12:283-293

19. Peremarti A, Twyman RM, Gomez-Galera S, Naqvi S, Farre G, Sabalza M, Miralpeix B, Dashevskaya S, Yuan D, Ramessar K, Christou P, Zhu C, Bassie L, Capell T (2010) Promoter diversity in multigene transformation. Plant Mol Biol 73:363-378

20. Potenza C, Aleman L, Sengupta-Gopalan C (2004) Targeting transgene expression in research, agricultural, and environmental applications: promoters used in plant transformation. In Vitro Cell Dev Biol Plant 40:1-22
21. Prasad AM, Sivanandan C, Resminath R, Thakare DR, Bhat SR, Srinivasan R (2005) Cloning and characterization of a pentatricopeptide repeat protein-encoding gene $(L O J)$ that is specifically expressed in lateral organ junctions in Arabidopsis thaliana. Gene 353:67-79

22. Prost E, Moore DD (1986) CAT vectors for analysis of eukaryotic promoters and enhancers. Gene 45:107-111

23. Resminath R, Prasad AM, Thakare DR, Sivanandan C, Bhat SR, Srinivasan R (2005) Promoter trapping in Arabidopsis using T-DNA insertional mutagenesis. J Plant Biochem Biotechnol $14: 1-8$

24. Saha D, Kumar V, Bhat SR, Srinivasan R (2011) Characterization of upstream sequences of the $L O J$ gene leads to the identification of a novel enhancer element conferring lateral organ junction specific expression in Arabidopsis thaliana. Plant Mol Biol Rep 29:265-277

25. Singer SD, Cox KD, Liu Z (2011) Enhancer-promoter interference and its prevention in transgenic plants. Plant Cell Rep 30:723-731

26. Sharma Y, Cheung U, Larsen EW, Eberl DF (2002) PPTGAL, a convenient Gal4 P-element vector for testing expression of enhancer fragments in drosophila. Genesis 34:115-118

27. Srinivasan R, Saha D (2010) Promoter trapping in plants using T-DNA mutagenesis. In: Jain SM, Brar DS (eds) Molecular techniques in crop improvement. Springer, New York, pp 545577

28. Szabados L, Charrier B, Kondorosi A, de Bruijn FJ, Ratet P (1995) New plant promoter and enhancer testing vectors. Mol Breeding 1:419-423

29. Weterings K, Schrauwen J, Wullems G, Twell D (1995) Functional dissection of the promoter of the pollen-specific gene NTP303 reveals a novel pollen-specific, and conserved cis-regulatory element. Plant J 8:55-63 